\title{
Poster Abstracts
}

\section{Agricultural Production and Food Systems}

\section{P1 Assessing Food Offerings Among Emerging Food Outlets in Northern Arkansas}

Bryan Mader, DPH, MPH, CHES, bmader@uaex.edu, University of Arkansas Cooperative Extension Services, 2301 South University Ave, Little Rock, AR, 72204

Background: Limited study of the availability of food in nontraditional food stores (Pharmacy and convenience stores) has left a gap in the understanding of the food environment in Arkansas. Recent corporate action among nontraditional food stores suggests that consumers are looking to these stores as sources of healthier food.

Objective: The question that guided this work asked, "Are there differences in food offerings among non-traditional food outlets, such as CVS and Walgreens, and how do those offerings compare to the offerings of convenience stores, in Arkansas?" Understanding differences among these food store types may be useful for subsequent efforts to improve equitable access to healthy food across Arkansas.

Study Design, Setting, Participants: This study used an observational design to assess offerings in non-traditional food outlets. The Nutrition Environment Measures Survey (NEMS) assessment tool was used to document and score the availability of food products in 54 stores in the northern half of Arkansas, including 27 pharmacy stores and 27 convenience stores within a 2.5 mile radius. Measurable Outcome/Analysis: The NEMS tool gathered availability data across 13 measures, including: fruit, vegetables, dairy products, meat products, frozen dinners, baked goods, bread, beverages, chips and snacks, and cereal.

Results: Pharmacy stores had a higher mean availability score $(\overline{\mathrm{X}}=19.0)$ than convenience stores $(\overline{\mathrm{X}}=7.2)$. A paired sample $t$ test indicated that the difference in mean availability score between the store types was not likely due to chance $[\mathrm{t}(26)=16.01, P<.001]$.

Conclusions: These findings suggest that pharmacy stores offer a healthier supply of foods as compared to convenience stores. Nontraditional food stores may be important intervention sites as consumers look to these stores for an increasing proportion of their food purchases. Further study may help to uncover differences in rural and urban food supply in Arkansas, and may have positive consequences for better understanding best practices for increasing access to food across the state.

Funding: None.

\section{P2 Benefits of Farmers' Markets for Food Access: Results from a Needs Assessment in a Rural Southern County}

Karen Franck,PhD, kfranck@utk.edu, University of Tennessee, 2621 Morgan Circle, Knoxville, TN, 37996; Kristen Johnson, PhD, RDN, University of Tennessee; Soghra Jarvandi, $P h D, M D$, University of Tennessee
Background: For a Centers for Disease Control and Prevention obesity prevention grant, a needs assessment was conducted in a rural Southern county with $40 \%$ adult obesity. The purpose of the grant was to reduce adult obesity rates through community interventions designed to improve access to healthy foods.

Objective: The objective was to identify barriers and facilitators for access to healthy foods.

Study Design, Setting, Participants: Adult county residents were recruited to complete surveys and participate in focus groups. Professionals were recruited for key informant interviews.

Measurable Outcome/Analysis: The survey included questions about access to healthy foods such as local availability and gardening. Descriptive statistics were used to examine survey data. Focus group and interview questions included perceived availability of healthy foods. Focus groups and interviews were transcribed verbatim. Two researchers coded the transcripts and developed themes using inductive content analysis.

Results: In summary, 1,085 surveys, 9 focus groups with 61 adults, and 25 interviews were completed. Focus group participants identified the county's 2 Farmers' Markets as community assets for healthy food, especially for residents who lived in the communities where the markets were located. A major theme was the importance of supporting local businesses such as Farmers' Markets and farmers. Over half of survey respondents (55\%) reported buying fruits and vegetables at these markets. There were differences in reasons why survey respondents and focus group participants did not shop at the Farmers' Markets. For survey respondents, the biggest barrier was that the markets were not open during the times they grocery shop (26.7\%). For qualitative interviews, participants identified lack of Electronic Benefit Transfer (EBT) acceptance, distance, and lack of variety as major barriers for not shopping at the Farmers' Markets.

Conclusions: These results indicate that Farmers' Markets can provide access to fruits and vegetables for small communities; however, economic and environmental barriers can limit market usage by limited-resource consumers.

Funding: Centers for Disease Control and Prevention.

\section{P3 Community Opportunity in New Jersey: The Role of Food Choice, Farm Viability, and Local Economy in a Sustainable Food Future}

Lauren B. Errickson, MS, lauren.errickson@rutgers.edu, Rutgers University, 88 Lipman Dr, New Brunswick, NJ, 08901; Ethan D. Schoolman, PhD, Rutgers University; Virginia Quick, PhD,RD, Rutgers University; Anthony Capece, Elijah's Promise; Graham E. Bastian, RDN, Rutgers University; Grace Chang, Rutgers University; Sarah Davis, None, Rutgers University; Jason Flatt, Elijah's Promise; Michelle Jimenez, Rutgers University; Michelle Jimenez, Rutgers University; Amanda Sie, Rutgers University

Background: Healthy food access can improve urban community health outcomes, and requires farm viability

Continued on page $S 17$ 